\title{
Multiple functionalization of fluorescent nanoparticles for specific biolabeling and drug delivery of dopamine $\dagger$
}

\author{
Maria Ada Malvindi, ${ }^{a b}$ Riccardo Di Corato, ${ }^{a c}$ Annalisa Curcio, ${ }^{d}$ Daniela Melisi, ${ }^{d}$ Maria Grazia Rimoli, ${ }^{d}$ \\ Claudia Tortiglione, ${ }^{e}$ Angela Tino, ${ }^{e}$ Chandramohan George, ${ }^{c}$ Virgilio Brunetti, ${ }^{b}$ Roberto Cingolani, ${ }^{c}$ \\ Teresa Pellegrino $^{a c}$ and Andrea Ragusa $* a c$
}

\author{
Received 12th July 2011, Accepted 27th September 2011 \\ DOI: 10.1039/c1nr10797f
}

\begin{abstract}
The development of fluorescent biolabels for specific targeting and controlled drug release is of paramount importance in biological applications due to their potential in the generation of novel tools for simultaneous diagnosis and treatment of diseases. Dopamine is a neurotransmitter involved in several neurological diseases, such as Parkinson's disease and attention deficit hyperactivity disorder (ADHD), and the controlled delivery of its agonists already proved to have beneficial effects both in vitro and in vivo. Here, we report the synthesis and multiple functionalization of highly fluorescent $\mathrm{CdSe} / \mathrm{CdS}$ quantum rods for specific biolabeling and controlled drug release. After being transferred into aqueous media, the nanocrystals were made highly biocompatible through PEG conjugation and covered by a carbohydrate shell, which allowed specific GLUT-1 recognition. Controlled attachment of dopamine through an ester bond also allowed hydrolysis by esterases, yielding a smart nanotool for specific biolabeling and controlled drug release.
\end{abstract}

\section{Introduction}

The development of new strategies for conjugating bioactive molecules to fluorescent probes has always attracted much attention due to its potential impact in the nanomedicine field. The advent of fluorescent semiconductor nanocrystals, both quantum dots (QDs) and quantum rods (QRs), has boosted research in this direction due to their unique optical and electronic properties. ${ }^{1}$

The neurotransmitter dopamine (DA) is involved in a variety of signaling pathways. ${ }^{2}$ As altered levels of dopamine have been linked to different pathological conditions, such as Parkinson's disease and attention deficit hyperactivity disorder (ADHD), the possibility to modulate brain DA levels might hold great promise for therapeutic purposes. ${ }^{3}$ However, DA alone is not able to

${ }^{a}$ National Nanotechnology Laboratory of CNR-NANO, via per Arnesano km 5, 73100, Lecce, Italy

${ }^{b}$ Center for Biomolecular Nanotechnology (CBN) of Istituto Italiano di Tecnologia (IIT), Via Barsanti, 73010 Arnesano, Lecce, Italy

'Italian Institute of Technology, via Morego 30, 16163 Genova, Italy

${ }^{d}$ Department of Pharmaceutical and Toxicological Chemistry, School of Pharmacy, "Federico II" University, via Domenico Montesano 49, 80131, Naples, Italy

"Istituto di Cibernetica "E Caianiello" (ICIB) of National Research Council of Italy (CNR), Via Campi Flegrei 34, 80078 Pozzuoli, Naples, Italy

$\uparrow$ Electronic supplementary information (ESI) available: TEM images, absorption and emission spectra, $\zeta$-potential and DLS graphics, gel electrophoresis images, cyclic voltammograms, western blot and RT-PCR data. See DOI: 10.1039/c1nr10797f cross the blood-brain barrier (BBB) because of its hydrophobicity and the lack of a specific transporter. ${ }^{4}$ On the other hand, L-dopa is a dopamine precursor which is able to penetrate the central nervous system (CNS) before being converted to dopamine. ${ }^{5}$ L-Dopa is currently used for the treatment of Parkinson's disease, however, conversion of L-dopa to dopamine also occurs in peripheral tissues, causing unwanted side-effects and reducing the effective amount of prodrug that reaches the brain. In this regard, a galactosylated dopamine prodrug (GalDa) already proved that glucose is able to mediate the diffusion of DA across the BBB through the GLUT-1 transporters located on the membrane of brain capillary endothelial cells, both in vitro and in vivo. ${ }^{4,6}$ However, a deeper understanding of the DA release/ accumulation into target neurons would be of paramount importance, and the availability of a fluorescent adduct might provide novel clues for pharmacodynamics and pharmacodistribution studies.

The group of Nadeau already conjugated dopamine to fluorescent QDs. ${ }^{7}$ Their approach involved direct attachment of the neurotransmitter to mercaptosuccinic acid-capped $\mathrm{CdSe} / \mathrm{ZnS}$ QDs. Nevertheless, their system proved to be very efficient and useful for biosensing red-ox cellular events, and it could be also exploited as photosensitizer because of the electron transfer between the nanoparticle and the neurotransmitter. ${ }^{7,8}$

In this study, highly fluorescent core/shell CdSe/CdS QRs were synthesized and made water-soluble and highly biocompatible by intercalating an amphiphilic polymer and conjugating diamino PEG molecules. Reductive amination with lactose was exploited to form an outer shell of galactose, which can be recognized by 
the GLUT-1 carriers, over-expressed in many tumor cells. Succinyl dopamine (DS) was later conjugated through standard EDC chemistry to the sugar via an ester bond, which can be easily hydrolyzed by cellular enzymes, thus releasing the prodrug. This complex system mimics the dopamine prodrug prepared by Bonina et al. ${ }^{4 a}$ although its conjugation to fluorescent nanocrystals might lead to a deeper understanding of its behavior, both in vitro and in vivo.

To the best of our knowledge this is the first example of functionalization of highly fluorescent QRs with two different biomolecules, galactose and dopamine, each with a specific task. These multiple-functionalized fluorescent nanoparticles were studied in detail and the specific molecular mechanism of internalization of the bioconjugates was investigated in vitro.

\section{Results and discussion}

\section{Synthesis and characterization of the bioconjugated nanocrystals}

Asymmetric core/shell CdSe/CdS QRs were synthesized according to a previously reported procedure. ${ }^{9}$ This seeded-growth colloidal methodology already proved to yield highly monodisperse nanocrystals with improved optical properties compared to classical QDs and also to more recent $\mathrm{CdSe} / \mathrm{ZnS}$ QRs, which is an important achievement for biological applications. In fact, this new class of nanocrystals has already been successfully exploited as biological marker in a variety of applications, both in specific and non-specific targeting. ${ }^{1 d, 10}$

The QRs used in this study have a length of $35 \mathrm{~nm}$ and a diameter of $5 \mathrm{~nm}$, as observed by TEM analysis, an excitonic peak at $592 \mathrm{~nm}$ and a corresponding band-edge emission peak at $598 \mathrm{~nm}$ in the absorption and photoluminescence (PL) emission spectra, respectively (see Fig. S2 in the ESI $\dagger$ ).

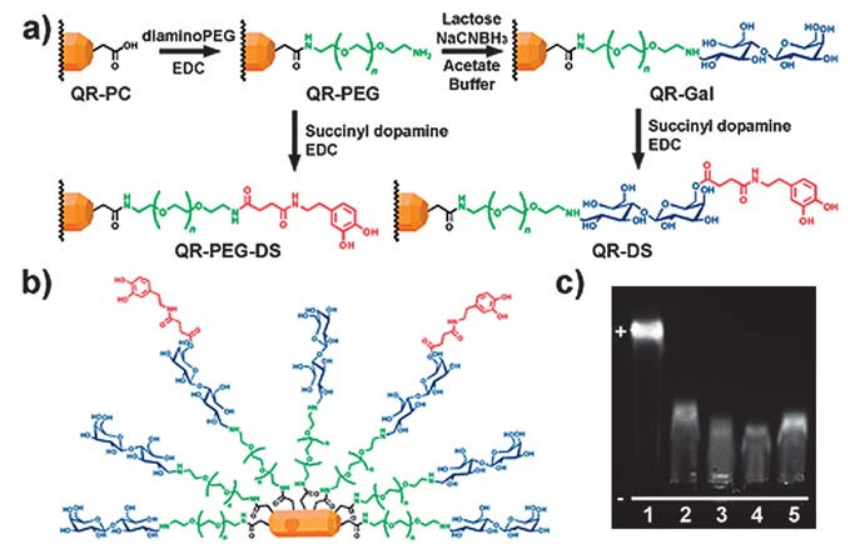

Fig. 1 (a) Synthetic scheme showing the various steps to get the dopamine bioconjugate. The polymer coated sample in water was reacted with diamino PEG through standard EDC chemistry. Reductive amination between the terminal PEG amino group and lactose yielded a galactose shell on the outer surface of the nanocrystal. Succinyl dopamine was finally attached to the carbohydrate through an ester bond, presumably with the less hindered hydroxylic group. (b) Schematic drawing of the final QR-DS nanoparticle. (c) Comparative gel electrophoresis migration patterns of the bioconjugated QRs after each synthetic step: (1) QR-PC; (2) QR-PEG; (3) QR-Gal; (4) QR-DS; and (5) QR-PEG-DS.
The synthetic strategy used to bio-functionalize the nanocrystals is depicted in Fig. 1a.

The as-synthesized nanocrystals were transferred into water by intercalating an octadecene polymer and cross-linking the outer anhydrides with a triamine, thus yielding carboxylic groups and amides on the outer surface of the nanocrystals. ${ }^{11}$ The watersoluble nanocrystals were purified by excess of polymer by ultracentrifugation and a diamino-PEG spacer was conjugated to the nanocrystals through standard EDC chemistry in order to improve biocompatibility. ${ }^{12}$ Lactose was then reacted with the terminal amino groups on the nanoparticles and the as-formed imine was reduced with sodium cyanoborohydride $\left(\mathrm{NaCNBH}_{3}\right)$. This reductive amination step yielded nanocrystals with terminal $\beta$-galactose units on the outer surface of the nanocrystal. Subsequent conjugation of succinyl dopamine was accomplished through standard carbodiimide chemistry, leading to the formation of an ester bond with the hydroxyl groups of the sugar. An ester bond was chosen since it is easily cleaved by enzymes, allowing the release of the dopamine inside the cell. In order to prove the specific role of the galactose residue, succinyl dopamine was also directly attached (by EDC chemistry) to the amino-PEG derivative. For simplicity, throughout the text the differently bioconjugated nanocrystals will be named QR-PC, QR-PEG, QR-Gal, QR-DS, and QR-PEG-DS, as also shown in Fig. 1a. A schematic drawing of the final QR-DS nanoparticle is presented in Fig. $1 \mathrm{~b}$.

The as-synthesized nanocrystals conserved the morphological characteristics of the original QRs and they were all perfectly soluble and stable for several months in biological media at $\mathrm{pH}$ 7.4, as observed by TEM analysis (see Fig. S1 in the ESI $\dagger$ ).

Gel electrophoresis analysis clearly showed the successful conjugation at each step of the synthetic route leading to the final compound (Fig. 1c). Lane 1 in Fig. 1c shows the migration pattern of the QRs after the polymer coating. As expected, addition of the amino-PEG coating caused a noteworthy retention of the nanocrystals due to the significant increase in the molecular weight and the change in the net charge of the system (Fig. 1c, lane 2). On the other hand, conjugation of galactose and succinyl dopamine (Fig. 1c, lanes 3 and 4, respectively) did not significantly affect the characteristics of the nanocrystal causing only a slight reduction in the corresponding migration patterns. The same trend was confirmed by $\zeta$-potential analysis in phosphate buffered saline (PBS, pH 7.4) (see Fig. S3 in the ESI $\dagger$ ). After polymer coating the nanocrystals showed a consistent negative net charge $(-34 \mathrm{mV})$ due to the high number of carboxylates at the surface. Reaction with diamino-PEG yielded amino groups at the surface while reducing at the same time the number of carboxylates, thus yielding a total net charge of -5 $\mathrm{mV}$. Again, as expected conjugation with galactose and succinyl dopamine shielded only slightly the positive charge on the amino groups, thus not affecting substantially the electronic surface properties of the bioconjugates and yielding a net charge of -10 and $-6 \mathrm{mV}$ for the two derivatives, respectively. The QR-PEGDS derivative resulted to have a $\zeta$-potential value of $-9 \mathrm{mV}$, consistent with the other bioconjugates.

The absorption and emission spectra of the various QRs in water did not change upon bioconjugation (see Fig. S2 in the ESI $\dagger$ ). On the other hand, variations could be observed in the corresponding photoluminescence (PL) quantum yield (QY). 
The QY of the as-synthesized QRs after polymer coating was measured to be $56 \%$. Addition of the amino-PEG coating lowered this value to $45 \%$, whereas galactose conjugation reduced the QY to $28 \%$. Covalent attachment of succinyl dopamine decreased further the QY to $18 \%$. Direct attachment of succinyl dopamine to the amino-PEG derivative yielded QRs with 10\% QY, probably because of the shorter distance between the neurotransmitter and the semiconductor nanocrystal in the absence of the galactose spacing. Electron transfer between dopamine and semiconductor QDs, which led to partial quenching of the nanocrystals, has already been reported in the literature. ${ }^{7,8}$ However, in this case the better optical properties of the starting $\mathrm{CdSe} / \mathrm{CdS} \mathrm{QRs}$, compared to traditional CdSe/ZnS QDs, led to higher values of QY, even after conjugation of dopamine.

\section{Electrochemical characterization}

Dopamine is a neurotransmitter and the electron transfer process between its reduced and oxidized state can be easily followed by electrochemical methods, such as cyclic voltammetry (CV), and in general, its oxidation potential is $V_{\text {ox }} \approx 0.5-0.8 \mathrm{~V}$ in PBS buffer solution on glassy carbon electrodes. ${ }^{13}$ Since quantitative information can be also obtained, we exploited this method to determine the average amount of dopamine bound to each type of nanocrystal.

When measuring the signal derived from a $1 \mu \mathrm{M}$ solution of QR-PEG-DS nanocrystals, an oxidative current corresponding to about $3 \mu \mathrm{M}$ dopamine could be assigned to the surface bound molecules (Fig. S4†, blue line). On the other hand, no signal was recorded when measuring bare QR-PEG nanocrystals (Fig. S4 $\uparrow$, orange line). However, the current profiles recorded in CVs suggest that, as expected, dopamine is covalently linked, and not physisorbed, to the QR-PEG nanoparticles, which hinder the diffusion of dopamine molecules to the electrode surface. This probably results in a severe diffusion limitation, unlike the diffusion of the free dopamine in solution, and poor redox behavior of the neurotransmitter when adhering to solid surfaces.

Diffusion effects influenced even more the measurement of the signal derived from a $1 \mu \mathrm{M}$ solution of QR-DS nanocrystals in which the redox behavior of the bound dopamine could not be followed clearly. This is probably due to the presence of the additional galactose shell which makes the nanocrystals even more "heavy" compared to the QR-PEG-DS nanoparticles. However, after in situ enzymatic cleavage, small anodic current corresponding to about $1.5 \mu \mathrm{M}$ dopamine could be observed (see Fig. S5 in the ESI $\dagger$ ). As expected, control experiments with QRGal nanocrystals, which do not bear any dopamine molecules, did not show any dopamine signal.

\section{Lectin binding assay}

To evaluate if galactose maintained its biological activity after conjugation to the nanoparticle, its affinity toward a Ricinus communis agglutinin, $\mathrm{RCA}_{120}$, a lectin specific for $\beta$-galactose units, was investigated. In fact, after reductive amination between lactose and the amino group on PEG, the nanoparticles present an outer shell composed of $\beta$-galactose moieties.

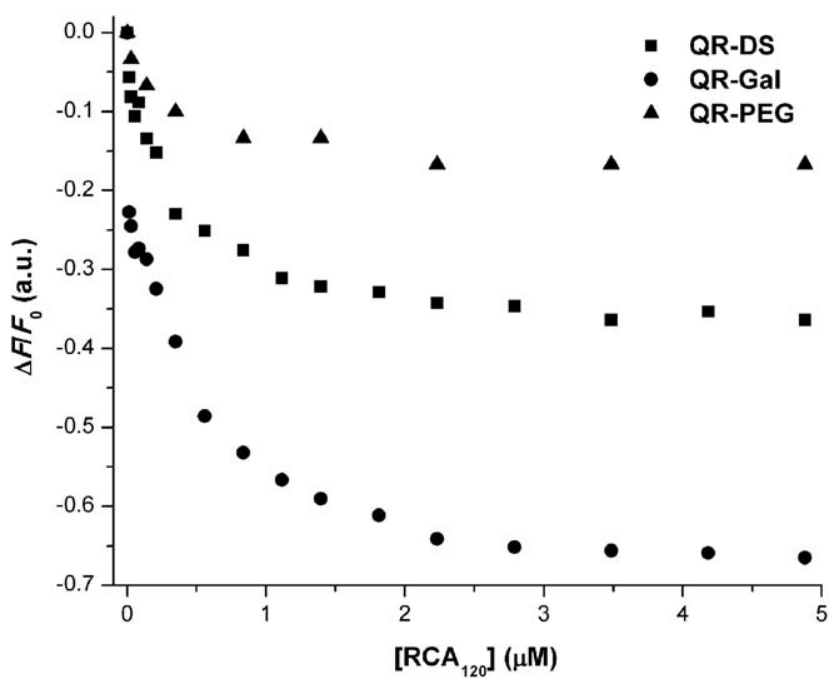

Fig. 2 Fluorescence intensity change $\left(\Delta F / F_{0}\right)$ of the nanocrystals upon addition of increasing amounts of lectin for QR-PEG (triangles), QRGal (circles), and QR-DS (squares).

Increasing amounts of $\mathrm{RCA}_{120}$ were added to the nanoparticles in PBS, thus causing a progressive quenching of the nanocrystal's fluorescence. The mechanism causing the fluorescence quenching still needs to be understood, although Robinson $e t$ al. proposed a radiationless deactivation pathway involving either an energy transfer or an electron transfer process upon complexation. ${ }^{14}$

The relative change in fluorescence intensity $\left(\Delta F / F_{0}\right)$ at $598 \mathrm{~nm}$ was plotted against the concentration of lectin added and the association constant $\left(K_{\mathrm{a}}\right)$ between the nanoparticles and the lectin was estimated by fitting the experimental data (Fig. 2). ${ }^{15}$

As expected, QR-Gal showed the highest affinity for the lectin ( $K_{\mathrm{a}}$ of the order of $10^{7} \mathrm{M}^{-1}$ ) because of the high number of terminal galactose units on its surface, inducing a reduction of more than a half of its original fluorescence intensity (Fig. 2, circles). The calculated $K_{\mathrm{a}}$ is about 3-4 orders of magnitude higher than the corresponding literature values for the $\beta$-Gal monomer, thus reflecting the multivalency of the nanoparticles and the cooperative effect of the ligands on their surface. ${ }^{15,16}$

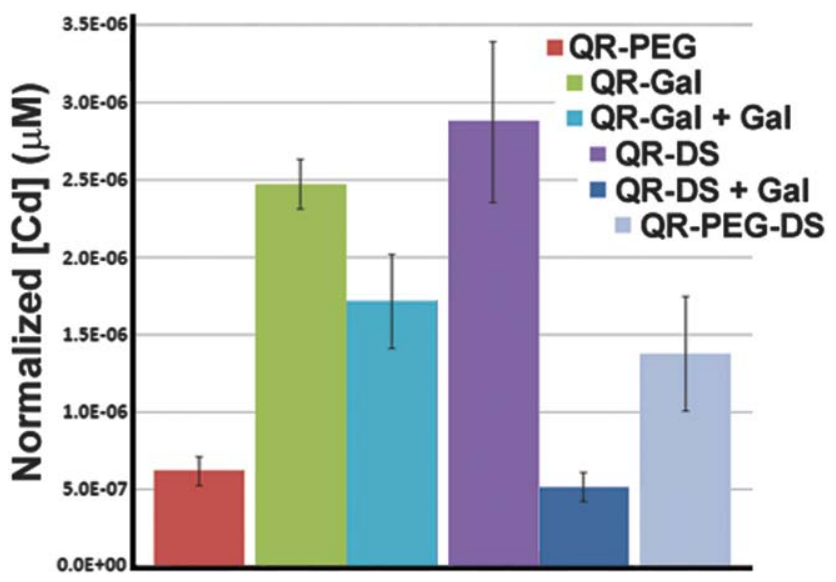

Fig. 3 Normalized internalization data for KB cells expressed as the amount of internalized cadmium (determined by ICP-AES) per cell as a function of the surface coatings. 
Conjugation of succinyl dopamine reduced the number of galactose moieties exposed to the environment, as confirmed by the lower amount of lectin required to reach saturation and which reduced the PL intensity of about $35 \%$ (Fig. 2, squares). As a control experiment, $\mathrm{RCA}_{120}$ was also added to QR-PEG (Fig. 2, triangles). However, in this case only little quenching of the PL intensity was observed (about 15\%), probably because of non-specific adsorption of the protein to the nanoparticle surface.
An excess of free galactose was also added after saturation of the QR-Gal solution, allowing a significant recovery of the original fluorescence by displacing the nanocrystals in a competitive manner (data not shown).

Dynamic light scattering (DLS) analysis of QR-Gal and QRDS upon addition of the lectin showed the formation of aggregates of about $120 \mathrm{~nm}$ in diameter, while subsequent additions led to the formation of a peak of about $14 \mathrm{~nm}$, corresponding to the excess of free lectin in solution (see Fig. S6 in the ESI $\dagger$ ).
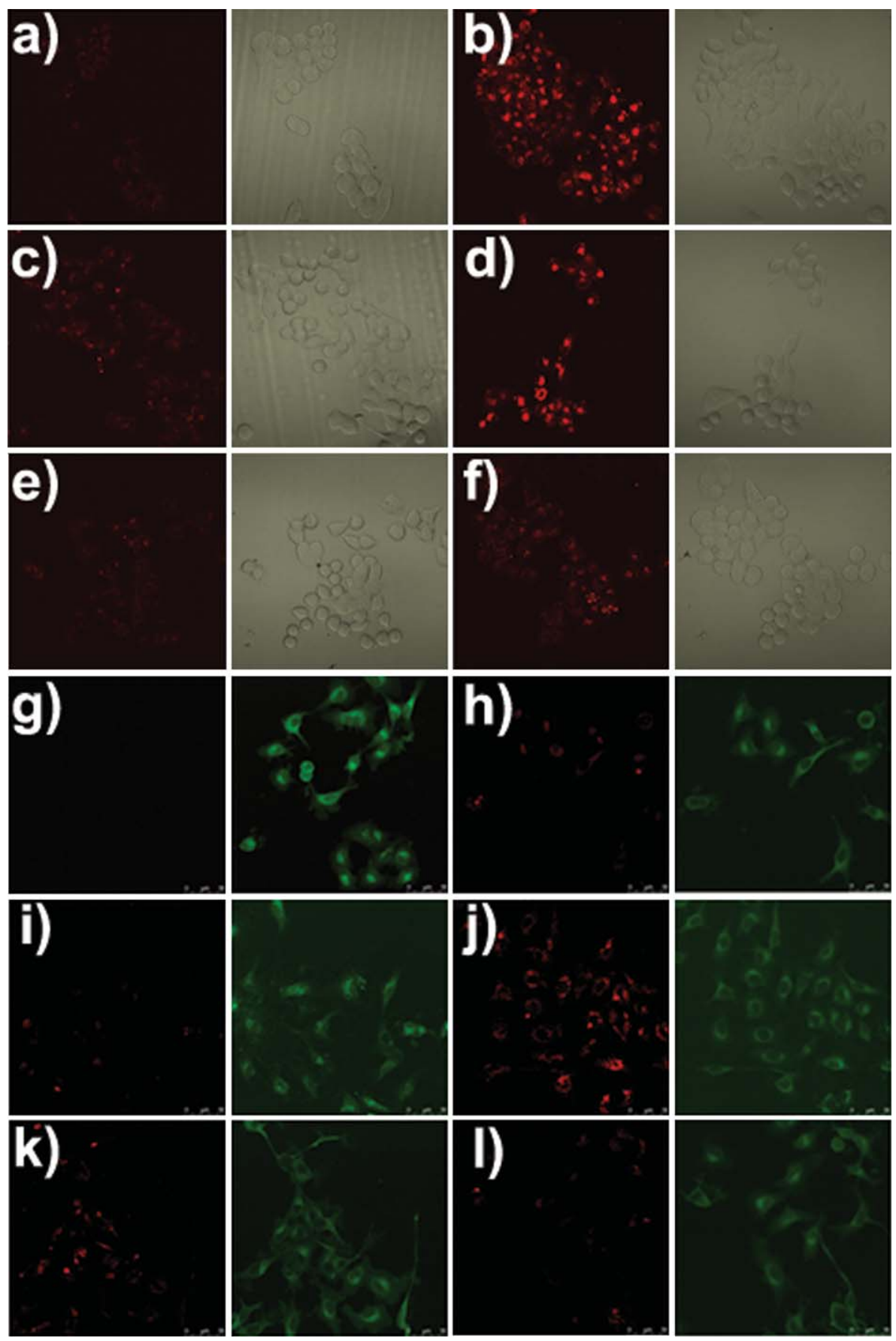

Fig. 4 Confocal microscopy images of KB cells treated with (a) QR-PEG; (b) QR-Gal; (c) QR-Gal + Gal; (d) QR-DS; (e) QR-DS + Gal; (f) QRPEG-DS; and of A549 cells treated with (g) QR-PEG; (h) QR-Gal; (i) QR-Gal + Gal; (j) QR-DS; (k) QR-DS + Gal; (l) QR-PEG-DS. For each letter, the panel to the left displays the $\mathrm{QR}$ fluorescence image while the one to the right displays either the corresponding transmission image (a-f) or the cell autofluorescence (g-l). 


\section{In vitro release of dopamine}

In order to test the real ability of the sample to release the dopamine, the enzymatic degradation of QR-DS nanocrystals by esterase from porcine liver was investigated in vitro. The hydrolysis should lead to the release of succinyl dopamine and to the recovery of $\mathrm{QR}-\mathrm{Gal}$ nanocrystals. Increasing amounts of porcine liver esterase $\left(0.5\right.$ and $\left.1 \mathrm{mg} \mathrm{mL}^{-1}\right)$ were added to a $20 \mathrm{nM}$ solution of nanoparticles in PBS and incubated at $37{ }^{\circ} \mathrm{C}$. The reaction was stopped after different incubation times and the electrophoretic migration pattern of the samples compared to those of the starting QR-DS and QR-Gal nanoparticles. Complete release of succinyl dopamine was observed after $24 \mathrm{~h}$ and very slight efficiency differences were observed between 2.5 and $5 \mathrm{mg} \mathrm{mL}^{-1}$ of esterase (see Fig. S7 in the ESI $\dagger$ ).

On the other hand, no hydrolysis was observed in PBS buffer solution ( $\mathrm{pH}$ 7.4) where the nanoparticles were stable even after several weeks.

\section{Cellular studies}

Cellular experiments were carried out to investigate the specific uptake of the bioconjugated nanocrystals. To quantify the number of nanoparticles taken up per cell, inductively coupled plasma atomic emission spectroscopy (ICP-AES) was used to determine the amount of internalized $\mathrm{Cd}$. Cell doping was always performed at a final total concentration of $100 \mu \mathrm{M}$ cadmium, equivalent to $5 \mathrm{nM}$ nanoparticle concentration. Cell numbers for all the samples and controls were measured after 24 hours and the cell growth rate for the samples exposed to the nanoparticles was found to be similar to the untreated control cells. QR-PEG nanocrystals were used as negative control. On the other hand, QR-Gal nanocrystals were used to show the specific GLUT-1 internalization. The glucose transporter GLUT-1 is responsible for the basal glucose uptake and is expressed on the plasma membrane of the cell. GLUT-1 has a high affinity for galactose and every culture cell line expresses GLUT-1. ${ }^{17}$ Competitive experiments, in which cell culture was saturated with free galactose before doping with the nanocrystals, were also performed to demonstrate the specific cell internalization.

KB cellular study. Human nasopharyngeal epidermal carcinoma (KB) cells overexpressing the GLUT transporter were used first.

ICP-AES results indicated drastically different internalization behavior depending on the ligand present at the surface of the nanocrystal (Fig. 3).

As expected, QR-PEG nanocrystals were internalized only in traces, probably through non-specific interaction. On the other hand, the QR-Gal bioconjugates showed high internalization values, suggesting that the galactose unit in the lactose residue is successfully recognized by the cell, supposedly through the GLUT-1 membrane transporter. This specific uptake was also confirmed by a competitive experiment in which the cell culture was saturated with free galactose $(1 \mathrm{mM})$ before adding the nanocrystals, thus saturating the GLUT-1 transporters at the cell surface.

A similar behavior was also observed after conjugation of succinyl dopamine to the sugar. Again, saturation of the culture medium with free galactose before addition of the nanocrystals reduced the internalization significantly, confirming the specific uptake. Furthermore, in this case the internalization was inhibited by free galactose even more significantly compared to the QR-Gal bioconjugate. Probably, this is due to the fact that there are less terminal galactose residues at the surface of the nanocrystal after the conjugation with succinyl dopamine, hence lower amounts of free galactose are required to inhibit its biological activity. Additionally, it seems that the dopamine residues at the surface of the QRs slightly increased the cellular uptake, indicating the possibility of specific recognition. Although this might seem surprising, there is already some evidence for the presence of dopamine transporters (DAT) in epithelial and submucosal tissues within nasal mucosa and they have been also exploited for the delivery of other dopamine prodrugs. ${ }^{18}$ However, western blot (WB) and reverse transcriptase-polymerase chain reaction (RT-PCR) molecular analyses revealed the absence of dopamine transporters on the surface of the KB cells used for these experiments, thus ruling out the possibility of specific DAT internalization (see Fig. S8 and S9 in the ESI for details $\dagger$ ).

As a further control experiment, cells doped with QR-PEGDS nanocrystals, which are missing galactose residues, showed a significantly reduced internalization compared to the QR-DS bioconjugates. Nevertheless, QR-PEG-DS nanoparticles were internalized somewhat more efficiently compared to the QRPEG nanocrystals, again confirming the apparent specific internalization through a dopamine receptor/transporter.

Fig. 4a-f show confocal microscopy images of KB cells loaded with the various $\mathrm{QR}$ bioconjugates.

As already seen by ICP, the compounds with the galactose residues (Fig. 4b and d) are internalized more efficiently compared to the others missing it (Fig. 4a and f) by clearly appearing brighter. On the other hand, minimal fluorescence signal was observed in the control experiments with free galactose in the cell medium (Fig. 4c and e).

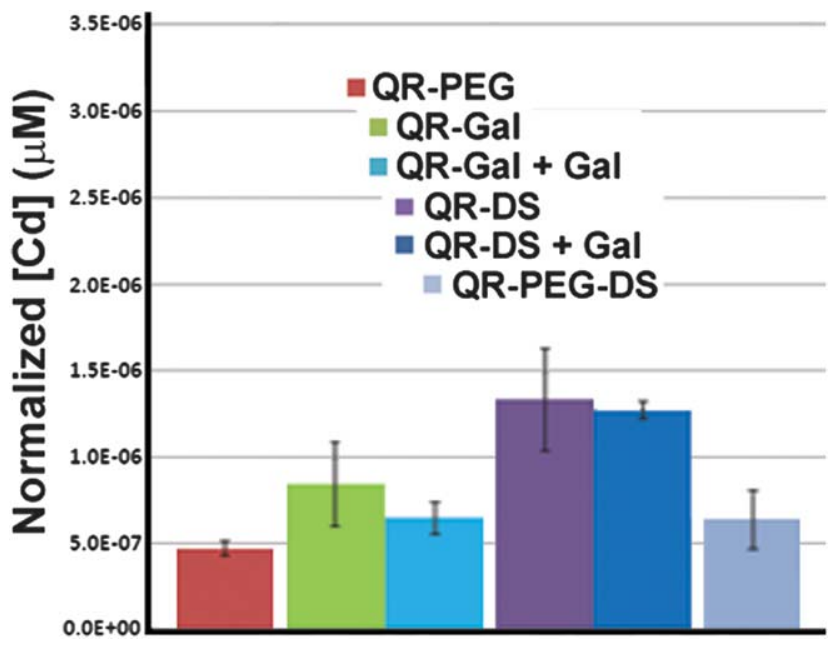

Fig. 5 Normalized internalization data for A549 cells expressed as the amount of internalized cadmium (determined by ICP-AES) per cell as a function of the surface coatings. The same scale as for KB cells was used to make easier the comparison of the internalization behavior between the two cell lines. 
A549 cellular study. In order to validate the theory of galactose recognition, human alveolar basal epithelial (A549) cells, which express the GLUT transporter to a minor extent compared to KB cells, were then tested. As expected, internalization experiments of the bioconjugated QRs, under the same conditions used with the KB cells, led to a much lower internalization of the nanocrystals of the carbohydrate-functionalized (QR-Gal and QR-DS) nanocrystals, as confirmed by ICP-AES analysis of the doped cells (Fig. 5).

Furthermore, the internalization was not inhibited significantly in the competitive assays with free galactose. Again, the QRs functionalized with dopamine (QR-DS and QR-PEG-DS)
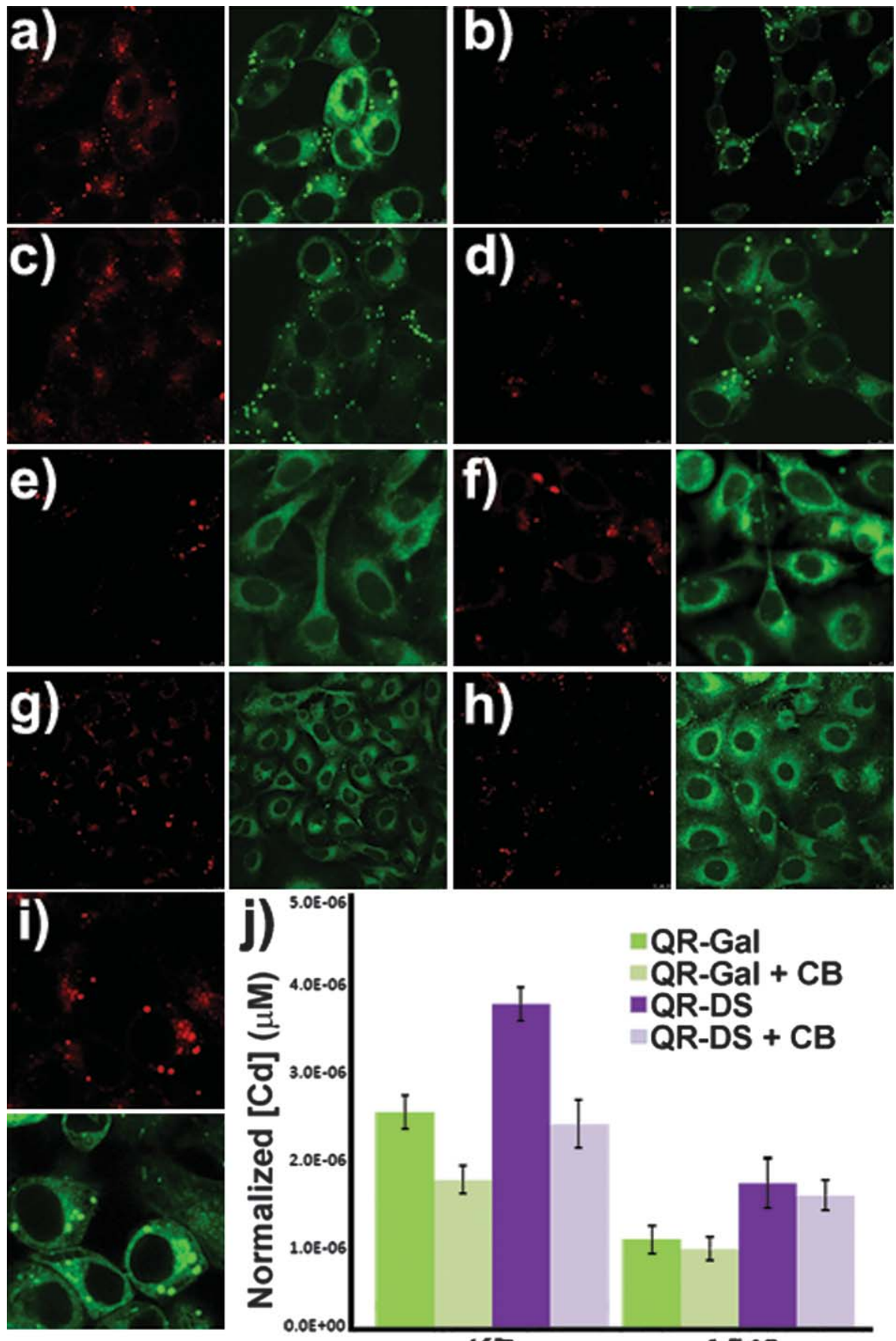

$0.06+00 L$ KB

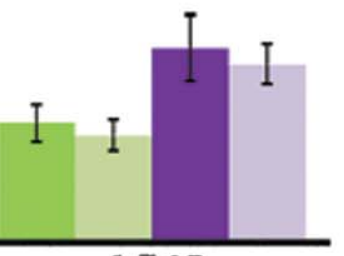

A549

Fig. 6 Confocal microscopy images of KB cells treated with (a) QR-Gal; (b) QR-Gal + CB; (c) QR-DS; (d) QR-DS + CB; and of A549 cells treated with (e) QR-Gal; (f) QR-Gal + CB; (g) QR-DS; (h) QR-DS + CB. For each letter, the panel to the left displays the QR fluorescence image while the one to the right displays the cell counterstained with Bodipy membrane dye. (i) QR fluorescence (top) and cell autofluorescence (bottom) magnification images of KB cells treated with QR-DS nanocrystals showing the formation of vesicles. (j) Normalized internalization data for KB and A549 cells expressed as the amount of internalized cadmium (determined by ICP-AES) per cell as a function of the surface coatings. 
were internalized a bit more compared to the corresponding ones without the bound neurotransmitter, but no sign of specific transporter was found by molecular analysis (see ESI for details $\dagger$ ). The same internalization trend was also observed by confocal microscopy (Fig. 4g-1).

Cytochalasin B inhibition assay. In order to rule out any doubt about the specific recognition of the bioconjugated nanocrystals by the GLUT-1 carrier, an inhibition test was carried out. Cytochalasin B (CB) is a well-known membrane-permeable mycotoxin that acts as a specific high-affinity inhibitor of glucose transport at the endofacial glucose binding sites of GLUTs. ${ }^{20}$ Both KB and A549 cells were treated with cytochalasin B for two hours before washing and addition of the nanocrystals. After 24 $\mathrm{h}$ the cells were washed and the nanocrystal internalization analyzed by ICP and confocal microscopy (Fig. 6).

As can be clearly observed, cytochalasin B successfully inhibited the internalization of the nanocrystals in $\mathrm{KB}$ cells. On the other hand, the same treatment on A549 cells did not affect significantly the already low internalization of the bioconjugated nanocrystals. Furthermore, magnification images of KB cells treated with QR-DS nanocrystals clearly showed the formation of vesicles inside the cells, typical of an endocytic internalization mechanism (Fig. 6i). All these data suggested that the nanocrystals presenting galactose moieties on their surface are being recognized by the GLUT-1 carrier on the outer membrane of the KB cells (and to a minor extent on that of the A549 cells). In a second step, the recognized nanocrystals are probably internalized through an endocytic pathway.

Cytotoxicity study. No sign of morphological damage to both cell lines (KB and A549) was observed upon treatment with the QR bioconjugates, showing the absence of toxicity. However, to investigate in more detail this aspect, MTT assay was also performed on both KB and A549 cells doped for 24 hours with the nanocrystals at $5 \mathrm{nM}$ concentration, equivalent to $100 \mu \mathrm{M} \mathrm{Cd}$ concentration (Fig. 7).

As expected, all the bioconjugated nanocrystals did not affect significantly the viability of the cells, showing their high biocompatibility. The galactose-conjugated nanocrystals (Fig. 7,

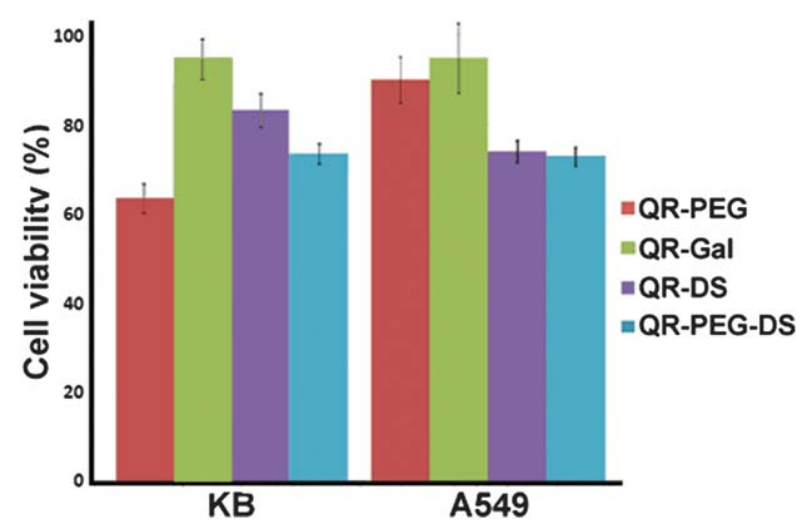

Fig. 7 MTT cytotoxicity assay performed on KB (left) and A549 (right) cells treated with the various nanocrystals. Cells were incubated with the NPs at $5 \mathrm{nM}$ concentration (equivalent to $100 \mu \mathrm{M} \mathrm{Cd}$ concentration) for 24 hours before analysis. green columns) showed cell viability values almost identical to control cells. However, this is not surprising considering that the outer shell of these NPs is made of carbohydrates, which are completely non-toxic.

\section{Conclusions}

Highly fluorescent $\mathrm{CdSe} / \mathrm{CdS}$ quantum rods were synthesized and successfully bio-functionalized. Galactose was introduced through a reductive amination step, while succinyl dopamine was subsequently coupled through an ester bond to the carbohydrate. The modified QRs showed excellent stability and the carbohydrate shell improved significantly the biocompatibility compared to the original nanocrystals, as shown by the MTT cytotoxicity assay. Moreover, the glyco-QRs maintaining their biological activity, as confirmed by a lectin binding assay, were selectively recognized by KB cells through the GLUT-1 transporter on the outer cellular membrane, and were internalized through an endocytic pathway, as the presence of vesicles was demonstrated. Therefore, the bio-functionalized glyco-QRs could find potential application in biodiagnostic devices or in in vivo cellular imaging.

Furthermore, the ability to release the dopamine residues was also demonstrated in vitro through hydrolysis mediated by esterase. The ability to preserve these properties also in vivo is now being tested in murine models and the results will be presented in due course. As one of the main challenges of brain targeting prodrugs is the possibility to detect and to quantify the drug in the brain, our approach joined in a single compound the advantages of the prodrug (i.e. the permeability for biological barriers, such as the BBB) and the optical properties of QRs (i.e. extraordinary brightness and stability) for ex vivo analysis. Thus, our bioconjugated QRs, able to selectively recognize specific cells and to deliver active dopamine into them, represent an innovative multifunctional nanoplatform which might be exploited for both imaging and therapeutic purposes.

\section{Experimental section}

\section{Chemicals}

All chemicals were used as received. Bis-(hexamethylene)triamine (\#14506), poly(maleic anhydride-alt-1-octadecene) (\#419117), sodium tetraborate decahydrate (\#S9640), boric acid (\#B1934), Tris-borate-EDTA buffer (\#T3913), sucrose (\#16104), $\quad N$-(3-dimethylaminopropyl)- $N^{\prime}$-ethylcarbodiimide hydrochloride (EDC, \#E7750), $\beta$-lactose (Lact, \#L3750), D(+)-galactose (Gal, \#112593), sodium cyanoborohydride $\left(\mathrm{NaCNBH}_{3}, \# 156159\right)$, dopamine hydrochloride (\#H8502), lectin from Ricinus communis (castor bean) agglutinin $\mathrm{RCA}_{120}$ (\#L7886), esterase from porcine liver (\#E2884), cytochalasin B from Drechslera dematioidea (\#C6762), and 3-(4,5-dimethyl-2thiazolyl)-2,5-diphenyl-2 $H$-tetrazolium bromide (MTT salt, \#135038) were purchased from Sigma-Aldrich. Diamine-PEG 897 (\#06703) was purchased from Fluka. Agarose (D-1 low EEO, \#C1349) was purchased from Eppendorf. Ultrapure grade water with a conductivity of $18.2 \mathrm{M} \Omega \mathrm{cm}$ was used in all experiments. Succinyl dopamine was synthesized in the laboratory of Prof. Rimoli, by reaction between dopamine and succinic anhydride, according to the procedure described by Bonina et $a$. $^{4 a}$ 
Synthesis of water-soluble nanocrystals and functionalization with diamino-PEG molecules

$\mathrm{CdSe} / \mathrm{CdS} \mathrm{QRs}$ with a length of $35 \pm 2 \mathrm{~nm}$ and a diameter of $5 \pm$ $0.4 \mathrm{~nm}$ were synthesized according to procedures developed by us and reported in a previous paper. ${ }^{9} \mathrm{CdSe} / \mathrm{CdS} \mathrm{QRs}$ emitting in the orange spectrum $\left(\lambda_{\max } 598 \mathrm{~nm}\right)$ were used to reduce the overlap with the autofluorescence signal of cells. QRs were transferred from the organic phase into water by adopting a polymer coating procedure by using poly(maleic anhydride-alt-1-octadecene). ${ }^{11}$ The excess of free polymer and other impurities was separated from the water-soluble nanocrystals through an ultracentrifugation step. ${ }^{11 b}$ A Beckman Coulter Optima LE-80K ultracentrifuge, equipped with a SW41 Ti rotor, was used at $33000 \mathrm{rpm}$ and on a continuous sucrose gradient. A $10-60 \%$ sucrose density range was used and the CdSe/CdS QRs were centrifuged for $2 \mathrm{~h}$. The surface carboxylic groups of the polymer were used to bind one of the amino moieties of the diamino-PEG molecules through the formation of an amide bond. ${ }^{12}$ Diamino-PEG molecules were introduced to increase the stability of the nanoparticles and to allow further surface functionalization.

\section{Nanocrystal conjugation to lactose}

Galactose-grafted nanocrystals were obtained by reacting the terminal amino groups of the PEG-functionalized nanocrystals with lactose through reductive amination. In a typical reaction, lactose $(5 \mu \mathrm{M})$ was added to a $0.1 \mu \mathrm{M}$ nanocrystal solution in acetate buffer ( $\mathrm{pH} 4.5)$ and $10 \mu \mathrm{M} \mathrm{NaCNBH}$. The mixture was sonicated at room temperature for $1.5 \mathrm{~h}$, after which the nanoparticles were purified by several washings with borate buffer (pH 9) through Amicon filters (30 000 MWCO).

\section{Nanocrystal conjugation to succinyl dopamine}

The lactose-functionalized nanocrystals were coupled to succinyl dopamine through standard EDC chemistry. In a typical experiment, $0.01 \mathrm{M}$ succinyl dopamine and $0.3 \mathrm{M}$ EDC were added to a $1 \mu \mathrm{M}$ nanocrystal solution in borate buffer $(\mathrm{pH} 9)$. The reaction mixture was vortexed for $1.5 \mathrm{~h}$ at room temperature, after which the nanocrystals were purified by several washings with borate buffer ( $\mathrm{pH} 9)$ through Amicon filters (30 000 MWCO).

\section{Gel electrophoresis characterization}

The samples were run on $2 \%$ agarose gel in TBE buffer for $1 \mathrm{~h}$ at 100 V. Prior to gel electrophoresis, a solution containing OrangeG and 30\% glycerol in gel-loading buffer corresponding to $20 \%$ of the sample volume was added to each sample. At the end of the run, the gel was observed under UV light, and a red filter was used to detect the fluorescence of the nanocrystals.

\section{Low-magnification TEM}

The samples were prepared by dropping a dilute solution of nanocrystals in water on carbon-coated copper grids (Formvar/ Carbon $300 \mathrm{Mesh} \mathrm{Cu}$ ). TEM images were recorded on a JEOL Jem1011 microscope operating at an accelerating voltage of $100 \mathrm{kV}$.

\section{Dynamic light scattering (DLS) and $\zeta$-potential measurements}

A Zetasizer Nano ZS90 (Malvern, USA) equipped with a 4.0 $\mathrm{mW} \mathrm{He}-\mathrm{Ne}$ laser operating at $633 \mathrm{~nm}$ and an Avalanche photodiode detector was used. Measurements were made at $25{ }^{\circ} \mathrm{C}$ in phosphate buffered solutions $(\mathrm{pH}$ 7.4) of the particles $(0.01 \mu \mathrm{M})$. The size distributions are by volume $(\%)$ while the $\zeta-$ potential measurements are by intensity. Each sample has been measured 5 times and the results analyzed by Malvern Instruments Ltd software.

\section{UV-vis absorption and photoluminescence spectra}

Spectra were recorded using a Varian Cary 300 UV-vis spectrophotometer and a Cary Eclipse spectrofluorimeter. Photoluminescence quantum yield measurements were performed adopting the gradient method and using Rhodamine $6 \mathrm{G}$ as reference fluorescent dye (excitation wavelength at $488 \mathrm{~nm}$ ). Briefly, solutions of Rhodamine $6 \mathrm{G}$ at different concentrations were prepared, and their optical densities at the excitation wavelength ranged from 0.01 to 0.1 to avoid self-absorption effects in the photoluminescence spectra; their absorption and PL spectra were recorded. Then, the optical densities (at $488 \mathrm{~nm}$ ) and the integrated fluorescence intensities of the solutions were plotted in a graph. The series of points was then interpolated with a straight line of slope $m \mathrm{R} 6 \mathrm{G}$ and intercept equal to zero. The same approach was adopted for all nanocrystals, yielding therefore for each type of nanocrystal an interpolation line of slope $m \mathrm{NC}$ and an intercept close to zero. The PL QY from each nanocrystal sample was then calculated using the following equation: $\mathrm{QY} \mathrm{NC}_{\mathrm{NC}}=\mathrm{QY}_{\mathrm{R} 6 \mathrm{G}} m_{\mathrm{NC}} / m_{\mathrm{R} 6 \mathrm{G}}\left(\eta_{\text {solvent }} / \eta_{\mathrm{ethanol}}\right)^{2}$, where $\mathrm{QY}_{\mathrm{R} 6 \mathrm{G}}$ is the QY of Rhodamine G6 (which is known from the vendor), and $\eta_{\text {solvent }}$ and $\eta_{\text {ethanol }}$ are the refractive indices of the solvents in which the nanocrystal sample and the dye are dissolved, respectively.

\section{Cyclic voltammetry analysis}

Cyclic voltammetry experiments were performed in a conventional three-electrode system using PARSTAT 2273 advanced potentiostat/galvanostat. Glassy carbon disk electrodes (GCE $1.5 \mathrm{~mm}$ radius) were polished to mirror-like finishing using diamond slurry $(0.05 \mu \mathrm{m})$ and used as working electrodes. A platinum $(\mathrm{Pt})$ wire as counter and silver/silver chloride $(\mathrm{Ag} / \mathrm{AgCl})$ saturated with $\mathrm{KCl}$ as a reference electrode were used. Degassed (purged with nitrogen) borate buffer solution served as the electrolyte. All CV experiments were performed at room temperature using sealed glass cells.

\section{Elemental analysis}

Elemental analysis was carried out by inductively coupled plasma atomic emission spectroscopy (ICP-AES) with a Varian Vista AX spectrometer. Samples were dissolved overnight in $1 \mathrm{~mL}$ of concentrated $\mathrm{HCl} / \mathrm{HNO}_{3} 3: 1$ (v/v), diluted to $5 \mathrm{~mL}$ with ultrapure water, and the resulting solution was directly analyzed.

\section{Lectin binding assay}

Increasing amounts of $\mathrm{RCA}_{120}$ were added to a $10 \mathrm{nM}$ solution of nanoparticles in PBS ( $\mathrm{pH}$ 7.4). The fluorescence emission 
spectra of the nanoparticles were recorded and the normalized PL decrease plotted as a function of the concentration of lectin added. The association constant $\left(K_{\mathrm{a}}\right)$ was derived from eqn (1):15

$$
\text { [Lectin] } F_{0} / \Delta F=\left[\text { Lectin] } F_{0} / \Delta F_{\max }+F_{0} / \Delta F_{\max } K_{\mathrm{a}}\right.
$$

where [Lectin] is the concentration of lectin added, $F$ is the fluorescence intensity, $F_{0}$ is the original PL intensity of the free nanoparticles, and $\Delta F_{\max }$ is the largest change in PL intensity after saturation with the lectin. The fluorescence titration curves were analyzed by the nonlinear hyperbolic curve-fitting method to obtain the corresponding association constants.

\section{In vitro release experiments}

Esterase from porcine liver $\left(0.5\right.$ and $\left.1 \mathrm{mg} \mathrm{mL}^{-1}\right)$ was added to a solution of the nanoparticles $(20 \mathrm{nM})$ in PBS and the mixture incubated at $37{ }^{\circ} \mathrm{C}$. Small aliquots were collected at different times and their electrophoretic migration was compared to those of the starting QR-DS and QR-Gal nanocrystals.

\section{Cell culture}

$\mathrm{KB}$ cells were grown at $37{ }^{\circ} \mathrm{C}$ in a $5 \% \mathrm{CO}_{2}$ atmosphere in RPMI1640 medium, supplemented with L-glutamine $(2 \mathrm{mM})$, penicillin (100 units per $\mathrm{mL}$ ), streptomycin $\left(100 \mu \mathrm{g} \mathrm{mL}^{-1}\right)$, and $10 \%$ heatinactivated fetal bovine serum (FBS). A549 cells were routinely cultivated in high glucose DMEM medium with $50 \mu \mathrm{M}$ glutamine, supplemented with $10 \% \mathrm{FBS}, 100 \mathrm{U} \mathrm{mL}^{-1}$ penicillin, and $100 \mathrm{mg} \mathrm{mL}^{-1}$ streptomycin.

\section{Treatment of KB and A549 cells with the nanocrystals}

$\mathrm{KB}$ and A549 cells $\left(10^{5}\right)$ were seeded in each well of a 6-well plate. The solution was removed and the cells were washed with PBS three times, after which a fresh medium, containing the nanocrystal conjugates at $100 \mu \mathrm{M} \mathrm{Cd}$ concentration, was added to each well. For the competitive experiments free galactose $(1 \mathrm{mM}$ final concentration) was added 30 minutes before adding the nanocrystal conjugates.

\section{Confocal microscopy imaging of cells}

Confocal microscopy images were recorded on an Olympus FV-1000 microscope for KB cells and on a Leica TCS-SP5 AOBS microscope for A549 cells. Both instruments were equipped with an argon laser source (excitation at $488 \mathrm{~nm}$ ) with a DM405/488-type dichroic filter and an acquisition window at $598 \pm 20 \mathrm{~nm}$. Imaging was performed on fixed cells after $24 \mathrm{~h}$ of incubation at $37^{\circ} \mathrm{C}$.

\section{Determination of the intracellular uptake of $\mathrm{Cd}$ and nanoparticles}

To estimate the intracellular $\mathrm{Cd}$ concentration and hence the intracellular nanocrystal uptake, $10^{5}$ cells were seeded in $1 \mathrm{~mL}$ of medium in each well $(3.5 \mathrm{~cm}$ in diameter) of a 6-well plate. After $24 \mathrm{~h}$ of incubation at $37^{\circ} \mathrm{C}$, the medium was replaced with fresh medium containing the nanocrystals at $100 \mu \mathrm{M}$ Cd concentration. After $24 \mathrm{~h}$ of incubation at $37^{\circ} \mathrm{C}$, the medium was removed, the cells were washed three times with PBS ( $\mathrm{pH} 7.4)$, trypsinized, and counted using a cell-counting chamber. Then, the cell suspensions were digested using a $\mathrm{HCl} / \mathrm{HNO}_{3} 3: 1$ (v/v) solution, and the intracellular $\mathrm{Cd}$ concentration was measured by means of elemental analysis and normalized to the number of cells. To convert the intracellular $\mathrm{Cd}$ concentration to nanoparticle concentration, the procedure described by Deka and colleagues was employed. ${ }^{19}$ In detail, the average diameter and length of the nanoparticles were assessed via statistical analysis on TEM images. The average number of $\mathrm{Cd}$ atoms per nanoparticle was determined by building a structural model of the nanoparticle with the same geometrical parameters as determined by TEM. Then, knowing the average number of $\mathrm{Cd}$ atoms per nanoparticle and the total concentration of this species in solution, it is possible to determine the concentration of nanoparticles.

\section{Treatment of KB and A549 cells with cytochalasin B and the nanocrystals}

KB and A549 cells $\left(10^{5}\right)$ were seeded in each well of a 6-well plate. The solution was removed and the cells were washed with PBS three times. Cytochalasin B $(20 \mu \mathrm{M})$ was added to the growing media and incubated for two hours. Afterwards, the cells were washed and nanocrystal conjugates at $100 \mu \mathrm{M}$ Cd concentration were added to each well. After $24 \mathrm{~h}$ of incubation at $37^{\circ} \mathrm{C}$, the medium was removed, the cells were washed three times with PBS (pH 7.4) and analyzed by confocal microscopy imaging and ICP measurements. Confocal microscopy images were recorded on a Leica TCS-SP5 AOBS microscope. The instrument was equipped with an argon laser source (excitation at $488 \mathrm{~nm}$ ) with a DM405/488-type dichroic filter and an acquisition window at $598 \pm 20 \mathrm{~nm}$. Imaging was performed on fixed cells using the membrane dye Bodipy 500/510 $\mathrm{C}_{4}, \mathrm{C}_{9}(1 \mathrm{nM})$. ICP analysis was performed as reported above.

\section{Cytotoxicity assay}

The MTT viability assay was performed to estimate the cytotoxicity of the conjugated nanocrystals. ${ }^{21}$ Specifically, $5 \times 10^{4}$ cells were first seeded in each well of a 12-well plate, and after incubation at $37{ }^{\circ} \mathrm{C}$ for $24 \mathrm{~h}$, the medium was replaced with a fresh medium containing the nanocrystals at $100 \mu \mathrm{M} \mathrm{Cd}$ concentration. After additional $24 \mathrm{~h}$ of incubation at $37^{\circ} \mathrm{C}$, the medium was removed, and the cells were washed three times with PBS (pH 7.4). Then $1 \mathrm{~mL}$ of a solution containing $1 \mathrm{mg} \mathrm{mL}^{-1}$ of MTT dissolved in culture medium was added to each well. After a $3 \mathrm{~h}$ incubation at $37^{\circ} \mathrm{C}$, the MTT, reduced by the mitochondrial reductase of vital cells, formed a dark insoluble product, formazan. At this point, the medium was collected from each well, centrifuged, and the supernatant discarded. The dark pellet was dissolved in DMSO, leading to a violet solution, the absorbance of which at $570 \mathrm{~nm}$ was determined. The absorbance value can be correlated to the percentage of vital cells, by comparing the data of the doped cells with those of control cells (i.e., with no conjugates added to the medium).

\section{Acknowledgements}

The authors would like to thank Rosanna Mastria for providing the quantum rods and the European Commission for funding (Project Magnifyco, Contract NMP4-SL-2009-228622). 


\section{Notes and references}

1 (a) M. Bruchez, M. Moronne, P. Gin, S. Weiss and A. P. Alivisatos, Science, 1998, 281(5385), 2013-2016; (b) I. L. Medintz, H. T. Uyeda, E. R. Goldman and H. Mattoussi, Nat. Mater., 2005, 4(6), 435-446; (c) X. Michalet, F. F. Pinaud, L. A. Bentolila, J. M. Tsay, S. Doose, J. J. Li, G. Sundaresan, A. M. Wu, S. S. Gambhir and S. Weiss, Science, 2005, 307(5709), 538-544; (d) A. Quarta, A. Ragusa, S. Deka, C. Tortiglione, A. Tino, R. Cingolani and T. Pellegrino, Langmuir, 2009, 25(21), 12614-12622.

2 (a) C. Missale, S. R. Nash, S. W. Robinson, M. Jaber and M. G. Caron, Physiol. Rev., 1998, 78, 189-225; (b) M. Bentivoglio and M. Morelli, The Organization and Circuits of Mesencephalic Dopaminergic Neurons and the Distribution of Dopamine Receptors in the Brain, in Handbook of Chemical Neuroanatomy, ed. S. B. Dunnett, M. Bentivoglio, A. Bjorklund and T. Hokfelt, Elsevier, Amsterdam, 2005, pp. 1-107.

3 (a) J. A. Sergeant, H. Geurts, S. Huijbregts, A. Scheres and J. Oosterlaan, Neurosci. Biobehav. Rev., 2003, 27, 583-592; (b) S. V. Faraone, J. Sergeant, C. Gillberg and J. Biederman, World Psychiatr., 2003, 2, 104-113.

4 (a) F. P. Bonina, C. Puglia, M. G. Rimoli, D. Melisi, G. Boatto, M. Nieddu, A. Calignano, G. La Rana and P. de Caprariis, J. Drug Targeting, 2003, 11, 25-36; (b) A. Dalpiaz, R. Filosa, P. De Caprariis, G. Conte, F. Bortolotti, C. Biondi, A. Scatturin, P. D. Prasad and B. Pavan, Int. J. Pharm., 2007, 336, 133-139.

5 R. Djaldetti and E. Melamed, J. Neurol., 2001, 248, 357-362.

6 (a) L. A. Ruocco, D. Viggiano, A. Viggiano, E. Abignente, M. G. Rimoli, D. Melisi, A. Curcio, M. Nieddu, G. Boatto, E. Carboni, U. A. Gironi Carnevale and A. G. Sadile, Neuroscience, 2008, 152, 234-244; (b) L. A. Ruocco, D. Viggiano, M. Pignatelli, T. Iannaccone, M. G. Rimoli, D. Melisi, A. Curcio, S. De Lucia, E. Carboni, U. A. Gironi Carnevale, P. de Caprariis and A. G. Sadile, Behav. Brain Res., 2008, 187, 449-454.

7 (a) S. J. Clarke, C. A. Hollmann, Z. J. Zhang, D. Suffern, S. E. Bradforth, N. M. Dimitrijevic, W. G. Minarik and J. L. Nadeau, Nat. Mater., 2006, 5(5), 409-417; (b) S. J. Clarke, C. A. Hollmann, F. A. Aldaye and J. L. Nadeau, Bioconjugate Chem., 2008, 19(2), 562-568.

8 D. R. Cooper, N. M. Dimitrijevic and J. L. Nadeau, Nanoscale, 2010, 2, 114-121.

9 L. Carbone, C. Nobile, M. De Giorgi, F. Della Sala, G. Morello, P. Pompa, M. Hytch, E. Snoeck, A. Fiore, I. R. Franchini, M. Nadasan, A. F. Silvestre, L. Chiodo, S. Kudera, R. Cingolani, R. Krahne and L. Manna, Nano Lett., 2007, 7(10), 2942-2950.

10 (a) M. A. Malvindi, L. Carbone, A. Quarta, A. Tino, L. Manna, T. Pellegrino and C. Tortiglione, Small, 2008, 4(10), 1747-1755; (b)
C. Tortiglione, A. Quarta, M. A. Malvindi, A. Tino and T. Pellegrino, PLoS One, 2009, 4(11), e7698.

11 (a) T. Pellegrino, L. Manna, S. Kudera, T. Liedl, D. Koktysh, A. L. Rogach, S. Keller, J. Radler, G. Natile and W. J. Parak, Nano Lett., 2004, 4(4), 703-707; (b) R. Di Corato, A. Quarta, P. Piacenza, A. Ragusa, A. Figuerola, R. Buonsanti, R. Cingolani, L. Manna and T. Pellegrino, J. Mater. Chem., 2008, 18, 1991-1996.

12 A. Williams and I. T. Ibrahim, Chem. Rev., 1981, 81(6), 589-636.

13 (a) K. L. Adams, B. K. Jena, S. J. Percival and B. Zhang, Anal. Chem., 2011, 83(3), 920-927; (b) A. Berduque, R. Zazpe and D. W. M. Arrigan, Anal. Chim. Acta, 2008, 611(2), 156-162; (c) C. A. Martinez-Huitle, M. Cerro-Lopez and M. A. Quiroz, Mat. Res., 2009, 12(4), 375-384.

14 A. Robinson, J. M. Fang, P. T. Chou, K. W. Liao, R. M. Chu and S. J. Lee, ChemBioChem, 2005, 6(10), 1899-1905.

15 (a) Y. Miura, T. Ikeda and K. Kobayashi, Biomacromolecules, 2003, 4 (2), 410-415; (b) E. Nakata, T. Nagase, S. Shinkai and I. Hamachi, J. Am. Chem. Soc., 2004, 126(2), 490-495.

16 (a) M. Mammen, S.-K. Choi and G. M. Whitesides, Angew. Chem., Int. Ed., 1998, 37(20), 2754-2794; (b) D. A. Mann, M. Kanai, D. J. Maly and L. L. Kiessling, J. Am. Chem. Soc., 1998, 120(41), 10575-10582; (c) S. M. Dimick, S. C. Powell, S. A. McMahon, D. N. Moothoo, J. H. Naismith and E. J. Toone, J. Am. Chem. Soc., 1999, 121(44), 10286-10296; (d) T. K. Dam, R. Roy, D. Page and C. F. Brewer, Biochemistry, 2002, 41(4), 1359-1363; (e) C. R. Yonzon, E. Jeoungf, S. L. Zou, G. C. Schatz, M. Mrksich and R. P. Van Duyne, J. Am. Chem. Soc., 2004, 126(39), 1266912676; $(f)$ R. Robelek, L. F. Niu, E. L. Schmid and W. Knoll, Anal. Chem., 2004, 76(20), 6160-6165.

17 C. F. Burant, Facilitative Glucose Transport, in Principles of Medical Biology. Cell Chemistry and Physiology: Part III, ed. E. E. Bittar and N. Bittar, Jai Press Inc., Greenwich, Connecticut, 1996, vol. 4, p. 78.

18 (a) N. V. Chemuturi, J. E. Haraldsson, T. Prisinzano and M. Donovan, Life Sci., 2006, 79(14), 1391-1398; (b) N. V. Chemuturi and M. D. Donovan, Mol. Pharmaceutics, 2007, 4 (6), 936-942; (c) H. D. Kao, A. Traboulsi, S. Itoh, L. Dittert and A. Hussain, Pharm. Res., 2000, 17(8), 978-984.

19 S. Deka, A. Quarta, A. Falqui, M. G. Lupo, G. Lanzani, C. Giannini, R. Cingolani, T. Pellegrino and L. Manna, J. Am. Chem. Soc., 2009, 131, 2948-2958.

20 (a) P. G. V. Plagemann and R. D. Estensen, J. Cell Biol., 1972, 55, 179-185; (b) D. Basketter and W. Widdas, J. Physiol., 1978, 278, 389-401; (c) E. Cloherty, K. Levine and A. Carruthers, Biochemistry, 2001, 40, 15549-15561; (d) A. Salas-Burgos, P. Iserovich, F. Zuniga, J. Vera and J. Fischbarg, Biophys. J., 2004, 87, 2990-2999.

21 T. Mosmann, J. Immunol. Methods, 1983, 65, 55-63. 